\title{
Deficiencia de lipasa ácida lisosomal, una patología infrecuente
}

\author{
Catalina Gómez-Duarte, ${ }^{1}$ Víctor García, ${ }^{1}$ Verónica Botero, ${ }^{1}$ Ana Aristizabal, ${ }^{1}$ Gabriel Echeverri² y \\ Harry Pachajoa ${ }^{3}$ \\ ${ }^{1}$ Fundación Valle del Lili, Cali, ${ }^{2}$ Clínica de la Costa, Universidad de la Sabana, Chía; ${ }^{3}$ Universidad Icesi, Cali. Colombia
}

\section{Resumen}

El déficit de lipasa ácida lisosomal es una enfermedad genética poco prevalente, con alta morbimortalidad en niños y adultos. Se caracteriza por alteración del metabolismo lipídico que genera depósitos de ésteres de colesterol y triglicéridos en el organismo. La presentación clínica depende de la actividad enzimática. Se debe sospechar en pacientes con alteraciones lipídicas o alteraciones hepáticas después de descartar otros diagnósticos. Actualmente existe la opción de utilizar enzima recombinante, la cual puede mejorar los parámetros lipídicos y hepáticos, así como detener la progresión de la enfermedad. Es imperioso realizar el diagnóstico oportuno para iniciar de forma temprana el tratamiento específico, con el fin de prevenir la morbimortalidad. Se llevó a cabo revisión de la literatura en torno del déficit de lipasa ácida lisosomal, para orientar acerca de su fisiopatología, manifestaciones clínicas, diagnóstico y tratamiento.

PALABRAS CLAVE: Dislipidemia. Cirrosis. Enfermedad por depósito de esteres de colesterol. Deficiencia de lipasa ácida lisosomal. Esteatohepatitis. Hepatopatía criptogénica.

\begin{abstract}
Lysosomal acid lipase deficiency is a genetic disease with a low prevalence and high morbidity and mortality in children and adults. It is characterized by an alteration of lipid metabolism, which generates cholesterol and triglyceride esters deposits in the body. Its clinical presentation depends on enzymatic activity. This condition should be suspected in patients with lipid or liver alterations after ruling out other diagnoses. Currently, there is the option of using a recombinant enzyme, which can improve lipid and liver parameters, as well as disease progression. Establishing a timely diagnosis in order to initiate specific treatment early is imperative for the prevention of morbidity and mortality. The purpose of this work is to perform a review of the literature about lysosomal acid lipase deficiency and to guide about its pathophysiology, clinical manifestations, diagnosis and treatment.
\end{abstract}

KEY WORDS: Dyslipidemia. Cirrhosis. Cholesterol esters deposition disease. Lysosomal acid lipase deficiency. Steatohepatitis. Cryptogenic liver disease.

Correspondencia: Harry Pachajoa

E-mail: hmpachajoa@icesi.edu.co
Fecha de recepción: 12-12-2017

Fecha de aceptación: 18-07-2018

DOI: $10.24875 / G M M .18004024$
Gac Med Mex. 2019;155:291-297

Disponible en PubMed www.gacetamedicademexico.com 


\section{Introducción}

La lipasa ácida lisosomal (LAL; EC 3.1.13) es una glicoproteína que se expresa principalmente en macrófagos, hepatocitos, células intestinales y de Kupffer. Está codificada en el gen LIPA (MIM: 613497) localizado en el brazo largo del cromosoma 10q región 23.2-23.3. ${ }^{1-6}$ Esta glicoproteína actúa como enzima desempeñando un papel clave en el metabolismo del colesterol. ${ }^{6}$

Los esteres de colesterol y triglicéridos son trasportados en partículas de colesterol de lipoproteínas, especialmente las lipoproteínas de baja densidad (LDL) y las de muy baja densidad (VLDL) a los hepatocitos; ingresan a la célula mediante endocitosis mediada por receptores (LDL-R) hasta el lisosoma en donde son hidrolizados por LAL a colesterol libre y ácidos grasos libres. ${ }^{1-6} \mathrm{El}$ colesterol libre inhibe la acción de la 3-hidroxi-3-metil-glutaril-CoA reductasa (HMG-CoA) impidiendo la formación de novo de colesterol, la activación de proteínas que se unen a elementos reguladores de esteroles y los factores de transcripción responsables de la activación de los genes de síntesis del colesterol. ${ }^{6}$ El colesterol desempeña un papel importante como precursor en la síntesis de esteroides y ácidos biliares y como componente de las membranas celulares. ${ }^{6}$

El déficit de esta enzima (LAL-D; MIM:278000) resulta en disminución de la hidrólisis y acumulación de esteres de colesterol y triglicéridos en los lisosomas del hígado, vasos sanguíneos, bazo, glándulas adrenales, sistema hematopoyético, ganglios linfáticos y tracto gastrointestinal. Además, se asocia con reducción de la generación de colesterol libre y ácidos grasos libres. ${ }^{1,3-10}$ La disminución de colesterol libre se traduce en aumento de la actividad de elementos reguladores de esteroles y de HMG-CoA, con el respectivo incremento en la síntesis de colesterol libre y ácidos grasos libres.,11 Por otra parte, hay aumento de la secreción de colesterol VLDL y de los niveles de LDL. ${ }^{11,12}$ La disminución de colesterol libre reduce la activación de los receptores $X$ hepáticos, la expresión del trasportador ABCA1 y el flujo de la apolipoproteína $A 1(A p o B 100)$ y el colesterol HDL plasmáti$\mathrm{CO}^{12,13}$ (Figura 1).

EI LAL-D es una enfermedad huérfana, con un patrón de transmisión autosómico recesivo., 411,14,15 Se han identificado más de 50 mutaciones según la Human Gene Mutation Data Base; la mutación del sitio de splicing del exón 8-, E8SJM (c.894G>A; p.delS275
Tabla 1. Fenotipo de la deficiencia de lipasa ácida lisosomal

\begin{tabular}{l|l|l|}
$\begin{array}{l}\text { Manifestaciones } \\
\text { clínicas }\end{array}$ & $\begin{array}{l}\text { Forma temprana } \\
\text { (antes EW) }\end{array}$ & $\begin{array}{l}\text { Forma tardía } \\
\text { (CESD) }\end{array}$ \\
\hline $\begin{array}{l}\text { Inicio de la enfermedad } \\
\text { Hepatomegalia }\end{array}$ & $\begin{array}{l}\text { Primeros meses } \\
\text { de vida }\end{array}$ & $\begin{array}{l}\text { Infancia hasta } \\
\text { adultez }\end{array}$ \\
\hline Fibrosis hepática & Presente & Variable \\
\hline $\begin{array}{l}\text { Esplenomegalia } \\
\text { Malabsorción }\end{array}$ & Presente & Variable \\
\hline $\begin{array}{l}\text { Calcificaciones } \\
\text { adrenales }\end{array}$ & Marcada & Variable \\
\hline $\begin{array}{l}\text { Dislipidemia } \\
\text { Depósito de esteres de } \\
\text { colesterol }\end{array}$ & $\begin{array}{l}\text { Variable } \\
\text { EW = enfermedad de Wolman, CESD = enfermedad por depósito de esteres de } \\
\text { colesterol. }\end{array}$ & Variable \\
\hline
\end{tabular}

Q298), es la más frecuente y produce la deleción del exón 8 en el ARNm.4-6,11,14-17 Anteriormente, el LAL-D se dividía en dos fenotipos, en los que la actividad enzimática determina la presentación clínica: la forma infantil o enfermedad de Wolman y una forma menos severa conocida como enfermedad por depósito de esteres de colesterol: ;,3,4,6-10,18 en la primera, la actividad enzimática es casi nula, mientras que en la seguda hay actividad enzimática residual (1 a $12 \%$ de la normal). ${ }^{12}$ Actualmente esta denominación no se usa ya que se entiende que el LAL-D es una única entidad nosológica que cursa con diferentes fenotipos de presentación, iniciando precozmente y con rápida progresión o de inicio tardío y con progresión cambiante $^{11}$ (Tabla 1).

El objetivo de este trabajo es realizar una revisión de la literatura acerca del LAL-D y orientar acerca de su fisiopatología, manifestaciones clínicas, diagnóstico y tratamiento.

\section{Epidemiología}

La verdadera prevalencia del LAL-D no se conoce, sin embargo, se asume que oscila de 1 entre 40000 a 1 entre 350000 individuos según la población. ${ }^{5,11,16,19-21}$ En Alemania se estimó una prevalencia de 1 entre 50000 casos de enfermedad por depósito de esteres de colesterol y de 1 entre 350000 de casos de enfermedad de Wolman. ${ }^{16,20}$ Esta enfermedad se presenta con mayor frecuencia en individuos con ascendencia iraní-judía. Según Valles-Ayoub, la tasa en esta población es de alrededor de 1 entre 4200 personas, lo cual parece corresponder a una variante 

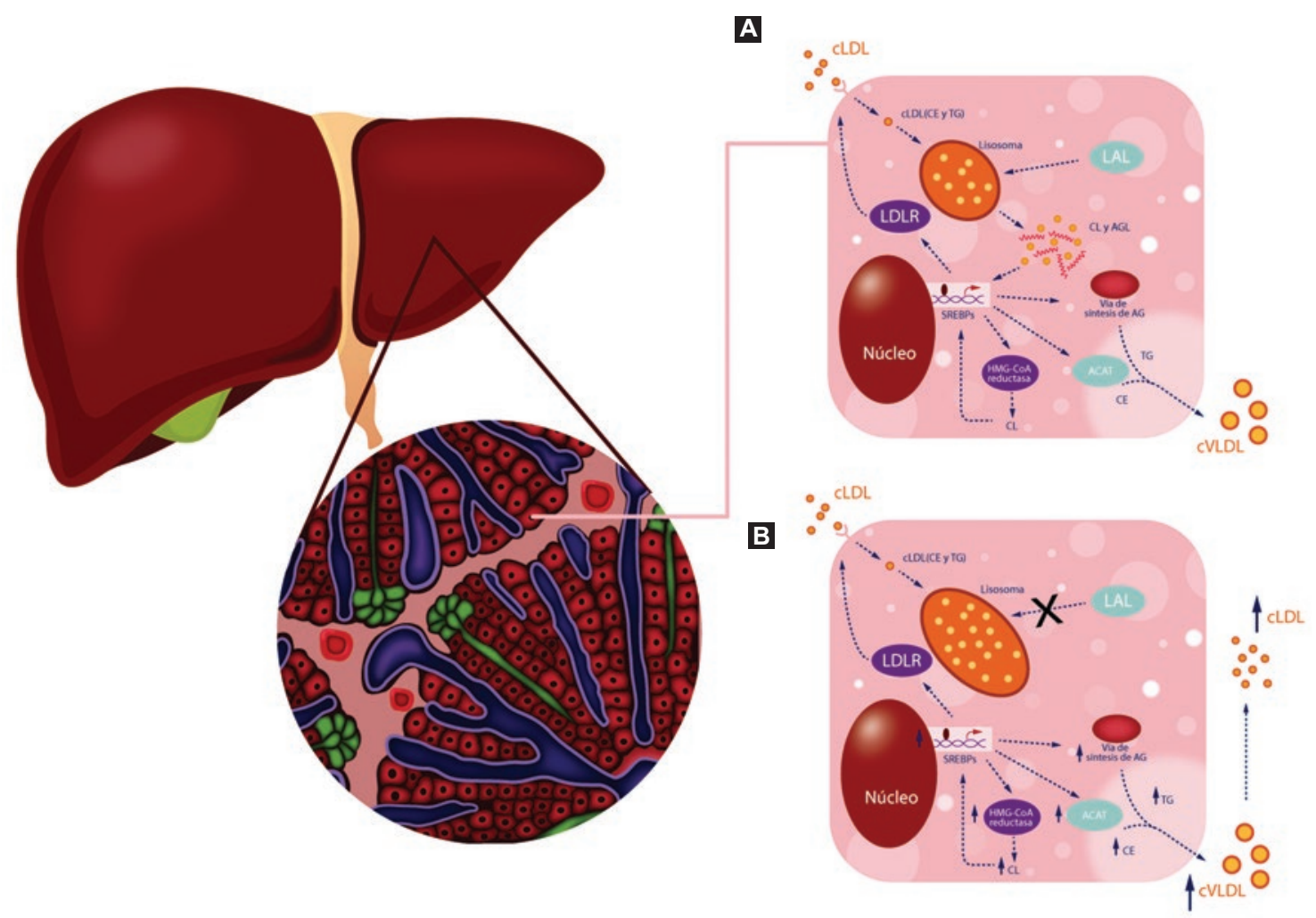

Figura 1. Esquema de la homeostasis del colesterol celular en A) individuos sanos y B) pacientes con deficiencia de lipasa ácida lisosomal. $A C A T=$ acetil-coenzima $A$ acetiltransferasa, $A G=$ ácidos grasos, $A G L=$ ácidos grasos libres, $C E=$ colesterol esterificado, $C L=$ colesterol libre, $c L D L=$ colesterol-low density lipoproteins, $c V L D L=$ colesterol very low density lipoproteins, HMG-CoA = hidroxi-metil-glutaril-coenzima $A$, $L A L=$ lipasa ácida lisosomal, $L D L R=$ low density lipoproteins receptor, $S R E B P=$ sterol regulatory element-binding proteins, $T G=$ triglicéridos.

mutacional asociada con la entidad (c.260G $>\mathrm{T}) .{ }^{22} \mathrm{La}$ mutación de c.894G>A (E8SJM) está presente en aproximadamente 50 a $70 \%$ de los pacientes con LAL-D tardía, los cuales tienen una actividad residual de la enzima. ${ }^{14,23}$ Por otro lado, las mutaciones de $\mathrm{C}$. 892C > T (E8SJM-3) y c.894p1G >A (E8SJMp1) se han detectado en LAL-D de inicio temprano. ${ }^{14,24}$

Esta enfermedad afecta por igual a uno y otro sexo y se ha reportado con mayor frecuencia en niños, con un pico de incidencia entre los tres y 15 años. ${ }^{12,25}$ Existen fenotipos con manifestaciones leves que no son diagnosticados debido a la falta de sospecha y acceso limitado a las pruebas diagnósticas confirmatorias, lo cual hace pensar que la prevalencia real de la entidad es mayor. No se han reportado estudios sobre la prevalencia de esta enfermedad en Colombia ni América Latina.

\section{Manifestaciones clínicas}

Las manifestaciones clínicas son variables, las más comunes incluyen dislipidemia, hepatomegalia y alteraciones hepáticas. ${ }^{4} \mathrm{EI}$ LAL-D de inicio temprano o enfermedad de Wolman se caracteriza por un cuadro clínico severo y fulminante que se expresa en las primeras semanas de vida. ${ }^{4-6,10,26-28}$ Clínicamente estos pacientes presentan diarrea crónica (esteatorrea), vómito, desnutrición crónica, malabsorción (debido a la esteatorrea y los episodios eméticos), falla en el crecimiento, hepatoesplenomegalia, compromiso hepático con desarrollo de fibrosis, colestasis y falla hepática. ${ }^{3-5,12,22,29}$ Asociado a esto, la mayoría de los pacientes presenta calcificación bilateral de las glándulas suprarrenales (50\%), lo que lleva a insuficiencia adrenal primaria, falla multiorgánica y muerte antes del primer año de vida. ${ }^{3-5,22,29}$

El LAL-D de inicio tardío o enfermedad por depósito de esteres de colesterol se caracteriza por un cuadro clínico más benigno y un amplio espectro de manifestaciones. ${ }^{6}$ Puede ser asintomático hasta la adultez, durante la cual se evidencia hepatomegalia ( $99 \%$ ) con esteatosis hepática microvesicular o mixta, esplenomegalia (74\%), alteraciones gastrointestinales y dislipidemia que genera aterosclerosis temprana con aumento del riesgo cardiovascular. . $-6,10,19,28-31^{\mathrm{Si}}$ el curso natural de la enfermedad persiste, la hepatopatía crónica puede provocar falla hepática, con la consecuente necesidad de un trasplante hepático. ${ }^{3-5,10,19,28-30}$ 


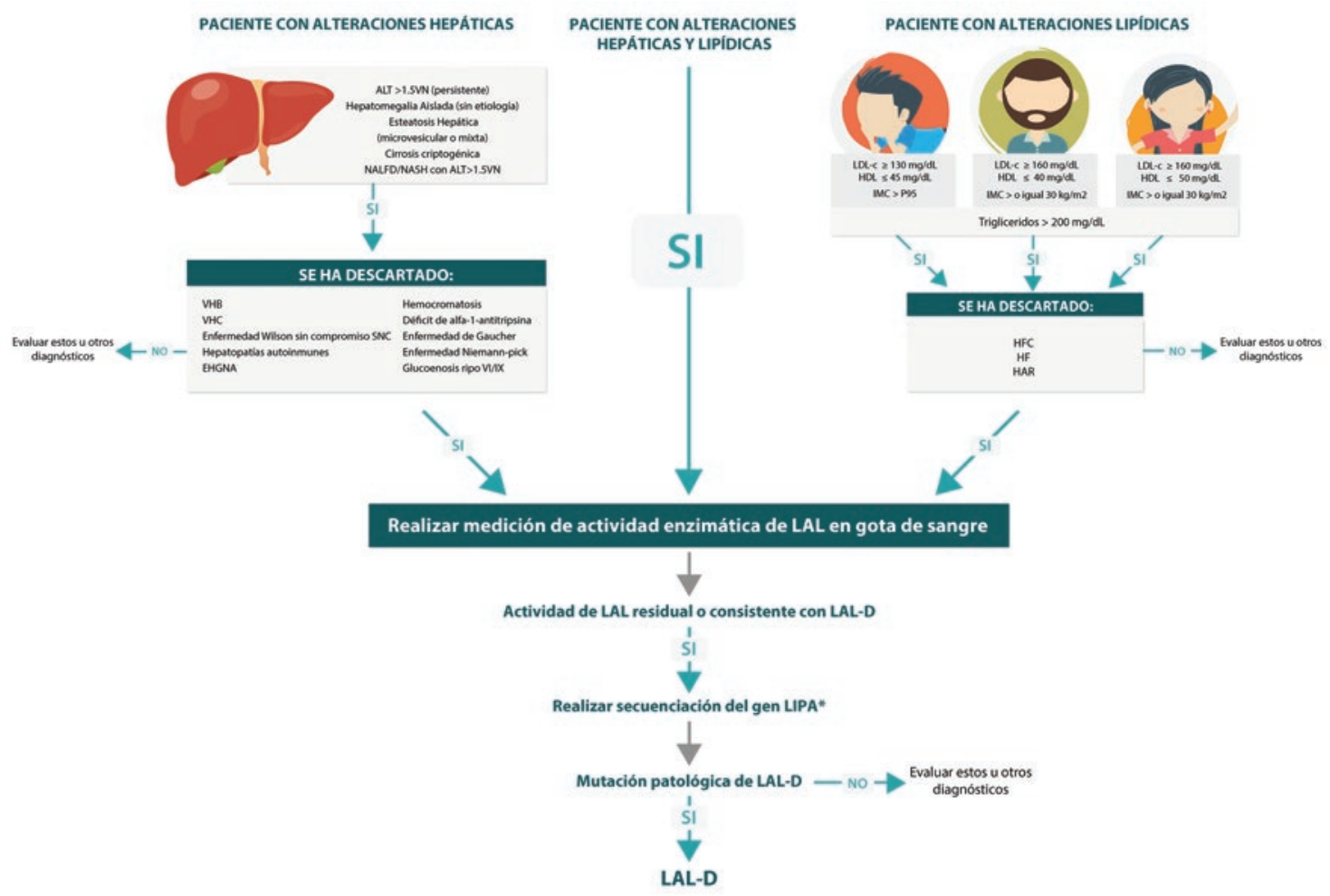

Figura 2. Guía para la sospecha clínica de lipasa ácida lisosomal. $A L T=$ alanina aminotransferasa, $C H D L=$ colesterol high density lipoproteins cholesterol, $C L D L=$ colesterol low density lipoproteins, $L A L-D=$ deficiencia de lipasa ácida lisosomal, EHGNA = enfermedad del hígado graso no alcohólico, $H A R=$ hipercolesterolemia autosómica recesiva, $H F=$ hipercolesterolemia familiar heterocigota, HFC = hiperlipidemia familiar combinada, $I M C$ = índice de masa corporal, $L A L=$ lipasa ácida lisosomal, $S N C=$ sistema nervioso central, VHB = virus de la hepatitis $B$, $\mathrm{VHC}=$ virus de la hepatitis $C, \mathrm{VN}=$ valor normal. ${ }^{*}$ Según disponibilidad.

Debido a la acumulación progresiva de esteres de colesterol y triglicéridos, en ambos espectros se generan alteraciones del perfil hepático que se caracterizan por aumento de los niveles de las transaminasas séricas (alanina aminotransferasa $[\mathrm{ALT}]$ y aspartato aminotransferasa [AST]), hiperbilirrubinemia y dislipidemia (aumento de las LDL, niveles bajos de las lipoproteínas de alta densidad [HDL] y aumento de triglicéridos). ${ }^{3-6,12,22,29}$

\section{Diagnóstico}

El reconocimiento del LAL-D es difícil debido a la baja prevalencia y al solapamiento con otras enfermedades. Asimismo, la ausencia de sospecha clínica puede llevar a errores en el diagnóstico, con la consecuente progresión de la enfermedad y retraso en el inicio del tratamiento.

En general se debe sospechar LAL-D en pacientes con hepatoesplenomegalia o alteraciones del perfil hepático o lipídico sin antecedentes familiares. El diagnóstico de esta patología se debe realizar acorde con la historia clínica, hallazgos sugestivos al examen físico y resultados del perfil lipídico. Reiner et al. sugieren que los pacientes con alteraciones en el perfil lipídico que no tengan antecedente familiar deben ser evaluados según los criterios diagnósticos; de cumplir con tres o más signos clínicos o paraclínicos, debe realizarse determinación de la actividad de la enzima en sangre ${ }^{4}$ (Figura 2).

Algunos recursos diagnósticos para apoyar el diagnóstico son la biopsia hepática y la resonancia magnética nuclear, la cual cuantifica el contenido de grasa hepática. ${ }^{12}$ Por otra parte, la biopsia hepática muestra hallazgos de la morfología hepática que pueden ser sugestivos más no confirmatorios, tales como esteatosis microvesicular con afectación de las células de Kupffer, fibrosis, cirrosis micronodular, histiocitosis en mar azul y cristales de esteres de colesterol.4,6,12,30,32,33 Otros hallazgos son acumulación de lípidos en las células reticuloendoteliales del bazo y macrófagos vacuolados con lípidos que infiltran la lámina propia con destrucción de la arquitectura del tejido gastrointestinal y la consiguiente disfunción en la actividad absorbente y enzimática de los enterocitos. ${ }^{6}$ En los exámenes de inmunohistoquímica se pueden usar 
marcadores lisosomales de catepsina $\mathrm{D}$, proteínas 1 y 2 asociadas con la membrana lisosomal (LAMP1 y LAMP2). ${ }^{32}$

El diagnóstico confirmatorio se realiza mediante la medición de la actividad enzimática en leucocitos de sangre periférica, fibroblastos de la piel o en gotas de sangre en papel filtro., ${ }^{5,12,34} \mathrm{El}$ análisis molecular con secuenciación genética de las regiones del gen LIPA se usa para identificar mutaciones puntuales, intrónicas profundas o reordenamientos..$^{12}$ Por otro lado, la técnica de gotas de sangre en papel filtro determina in vitro la actividad de LAL en presencia de sustratos (oleato, palmitato, etcétera) modificados con fluorocromo (4-metilumbeliferona), lo que permite cuantificar la cantidad de producto liberado por medio de espectroscopia de emisión o fluorimetría. . $^{6,12,35}$

Debido a que la presencia de otras lipasas en la sangre puede interferir con la medición, se utiliza un inhibidor de LAL Ilamado Lalistat $2^{\circledR}$ (Chemical Tools, South Bend, IN, Estados Unidos). ${ }^{35}$ La actividad de LAL es determinada comparando la actividad de la lipasa total con la actividad de lipasa en presencia del inhibidor; la diferencia entre las dos es atribuible a la enzima LAL. Valores reducidos de actividad enzimática de acuerdo con los rangos establecidos por el laboratorio se consideran diagnósticos de LAL-D. La secuenciación completa de la región codificante del gen LIPA permite la identificación de las mutaciones causales de la enfermedad. La mayoría de los pacientes son homocigotos o heterocigotos compuestos, ${ }^{4,12,35}$ sin embargo, algunos pueden tener mutaciones intrónicas $u$ otras variantes que pueden no ser detectadas en la secuenciación. Por tanto, el método de elección para el diagnóstico es la medición de la actividad enzimática y las pruebas moleculares se consideran complementarias. ${ }^{4}$

\section{Tratamiento}

En el pasado, el tratamiento consistía solo en la prevención de las complicaciones de la enfermedad. Se recomendaban cambios en el estilo de vida y manejo con hipolipemiantes (estatinas) para el manejo de la hipercolesterolemia. Las estatinas disminuyen los niveles de LDL, no obstante, no generan cambios en el desarrollo de la fibrosis hepática. 4,6,12,36,37

El trasplante de médula ósea se ha empleado en los casos de LAL-D con diagnóstico temprano para prevenir complicaciones cardiovasculares o en lactantes con LAL-D rápidamente progresiva, sin embargo, aún se necesitan más estudios para evaluar rigurosamente esta estrategia. ${ }^{3,4,6,12}$ Por su parte, el trasplante hepático es el tratamiento indicado para la falla hepática, debe realizarse en conjunto con el trasplante de médula ósea debido al riesgo de recurrencia de la enfermedad. Hasta el momento, el seguimiento de estos pacientes no permite obtener conclusiones sobre la eficacia de esta intervención en la LAL-D. 2,6,36,38,39

Actualmente se emplea la sustitución enzimática, con la que se busca restablecer los niveles fisiológicos de la enzima para así prevenir la acumulación de esteres de colesterol y triglicéridos al retrasar la progresión de la enfermedad. ${ }^{4,12}$ Se puede realizar la reposición con enzima recombinante de lipasa ácida lisosomal humana (rhLAL) o sebelipasa alfa recombinante (Kanuma ${ }^{\circledR}$, Alexion Pharmaceuticals), glicoproteína que contiene 378 aminoácidos de $53-55 \mathrm{kDa}$, seis sitios de glucosilación ligados- $N$ y es extraída de huevos de gallinas. .,40-42 $^{-10}$

Este fármaco ingresa a las diferentes células (células de Kupffer y hepatocitos, entre otros) por medio de los receptores de manosa-6-fosfato; una vez adentro, la enzima recombinante reemplaza a la $L A L$, hidrolizando esteres de colesterol y triglicéridos dentro del lisosoma para revertir sus efectos. . $^{3,12,43}$ Se ha utilizado en biomodelos con roedores, en los que se ha observado que corrige las anormalidades asociadas con esta condición: disminuye el contenido lipídico a nivel hepático, la esplenomegalia, los niveles de trasaminasas y la aterosclerosis. ${ }^{2,43-46}$ Se han realizado seis estudios para evaluar la eficacia y seguridad de este fármaco en lactantes con LAL-D (LAL-CL03 y LALCL08) y en pacientes pediátricos y adultos con LAL-D (LAL-CL01, LAL-CL04 y LAL-CL02).3,6,29,41,47 En ensayos clínicos en humanos se ha demostrado disminución de las enzimas hepáticas en sangre, del volumen hepático y de la grasa a nivel hepático, además, se ha conseguido mejoría de los niveles plasmáticos de lípidos (LDL y HDL), con aumento del colesterol-HDL y disminución del colesterol-LDL. 2,3,6,12,29,40,47 Asimismo, se realizaron ensayos clínicos I y II en adultos con LAL-D a quienes se les aplicó $0.35,1$ o $3 \mathrm{mg} / \mathrm{kg}$ endovenosos de sebelipasa-alfa durante siete días; se observó disminución de las transaminasas..$^{41,43}$

Los mismos pacientes del anterior estudio posteriormente fueron incluidos en una investigación de seguimiento de 52 semanas; todos iniciaron con el mismo manejo farmacológico con sebelipasa-alfa y a los 15 días se realizó ajuste a $103 \mathrm{mg} / \mathrm{kg}$. Se observó disminución del colesterol total, colesterol-LDL y triglicéridos, asociada con otros hallazgos como la reducción de la fracción grasa y del volumen hepático. ${ }^{41,48}$ Por 
último, en el estudio ARISE fase III se incluyeron pacientes pediátricos ( $\geq 4$ años) y adultos con LAL-D; de forma aleatorizada recibieron sebelipasa-alfa $(1 \mathrm{mg} / \mathrm{kg}$ cada dos semanas, $n=36)$ o placebo $(n=30)$ durante 20 semanas; entre los resultados se evidenció disminución de las transaminasas y del colesterol-LDL en el grupo manejado con sebelipasa-alfa comparado con el grupo que solo recibió placebo. ${ }^{29}$

El tratamiento con reposición enzimática (sebelipasa-alfa) en pacientes menores de seis meses de edad se inicia con $1 \mathrm{mg} / \mathrm{kg}$ una vez a la semana y se debe escalar a $3 \mathrm{mg} / \mathrm{kg}$ dependiendo del paciente. En aquellos con LAL-D de inicio tardío, la dosis es de $1 \mathrm{mg} / \mathrm{kg}$ cada 15 días y no se debe ajustar si son mayores de 65 años o si presentan falla renal o hepática. ${ }^{3,43}$ Los efectos adversos reportados no atribuibles al medicamento son náuseas, cefalea, diarrea y dolor abdominal. ${ }^{4,6,29,40,41}$

\section{Seguimiento}

Camarena et al. plantean que los pacientes deben tener un seguimiento clínico y paraclínico estricto (cada tres meses durante el primer año). En la primera visita se debe valorar el estado basal del paciente con biopsia hepática, imágenes diagnósticas y exámenes paraclínicos (hemograma, pruebas de coagulación, perfil lipídico y hepático). ${ }^{11}$ Posteriormente se debe evaluar su respuesta al tratamiento con valoración clínica y paraclínica seriada. En cuanto al seguimiento específico en los niños se debe monitorear el crecimiento y estado nutricional, mientras que en los adultos se debe realizar el seguimiento de acuerdo con la severidad de la enfermedad. ${ }^{49}$

\section{Diagnóstico diferencial}

El LAL-D presenta características clínicas que se pueden confundir con otras enfermedades, como la esteatosis hepática no alcohólica, la enfermedad hepática grasa no alcohólica, el síndrome metabólico, la hipercolesterolemia autosómica recesiva (MIM: 603813), la sitosterolemia (MIM: 210250) o la hipercolesterolemia familiar (MIM: 143890).4,5,11,50

En cuanto a las diferencias entre hipercolesterolemia familiar y LAL-D, la primera es una enfermedad genética de carácter autosómico dominante, es decir, que los pacientes deben tener antecedentes familiares de la enfermedad. Por otra parte, el perfil lipídico muestra niveles de LDL y HDL más altos en la hipercolesterolemia familiar., ${ }^{4,5}$ Por lo general, a diferencia de los pacientes con LAL-D, los pacientes con síndrome metabólico presentan obesidad. ${ }^{4}$ En cuanto a las diferencias entre enfermedad hepática grasa no alcohólica y LAL-D, los pacientes suelen presentar obesidad o sobrepeso y en la biopsia hepática los hallazgos son diferentes, en la mayoría se observa esteatosis macrovesicular. ${ }^{50}$

\section{Conclusiones}

El LAL-D es una enfermedad poco prevalente y subdiagnosticada, generada por mutaciones en el gen LIPA. En esta patología se afecta el metabolismo lipídico a nivel lisosomal y se caracteriza por la acumulación sistémica y progresiva de colesterol esterificado y triglicéridos. El fenotipo de presentación depende del grado de actividad enzimática, cuando es nula, el LAL-D se manifiesta tempranamente y de forma severa, con alta mortalidad al año de vida. La presentación tardía tiene un fenotipo heterogéneo: la mayoría de los pacientes desarrolla hepatopatía crónica y algunos, enfermedad cardiovascular precoz.

El diagnóstico representa un reto ya que las manifestaciones clínicas se solapan con las de otras enfermedades. Se debe sospechar LAL-D ante alteraciones hepáticas o lipídicas persistentes sin causa clara. El diagnóstico precoz es esencial para el manejo con tratamiento enzimático sustitutivo, el cual ha demostrado mejorar los parámetros lipídicos y hepáticos, así como la supervivencia de los lactantes con enfermedad rápidamente progresiva. ${ }^{51}$ No obstante, se estima que la prevalencia real de este trastorno esta subestimada ante la falta del conocimiento y la limitación de pruebas diagnósticas para esta enfermedad, por lo cual se torna necesaria la realización de estudios de prevalencia en Latinoamérica.

\section{Bibliografía}

1. Aslanidis C, Ries S, Fehringer P, Büchler C, Klima H, Schmitz G. Genetic and biochemical evidence that CESD and Wolman disease are distinguished by residual lysosomal acid lipase activity. Genomics. 1996; 33:85-93.

2. Rader DJ. Lysosomal acid lipase deficiency: a new therapy for a genetic lipid disease. N Engl J Med. 2015;373:1071-1073.

3. Frampton JE. Sebelipase alfa: a review in lysosomal acid lipase deficiency. Am J Cardiovasc Drugs. 2016;16:461-468.

4. Reiner Ž, Guardamagna O, Nair D, Soran H, Hovingh K, Bertolini S, et al. Lysosomal acid lipase deficiency: an under-recognized cause of dyslipidaemia and liver dysfunction. Atherosclerosis. 2014;235:21-30.

5. Wierzbicka-Rucińska A, Jańczyk W, Ługowska A, Lebensztejn $D$, Socha P. Diagnostic and therapeutic management of children with lysosomal acid lipase deficiency (LAL-D). Review of the literature and own experience. Dev Period Med. 2016:20:212-215.

6. Su K, Donaldson E, Sharma R. Novel treatment options for lysosomal acid lipase deficiency: critical appraisal of sebelipase alfa. Appl Clin Genet. 2016;9:157-167. 
7. Grabowski G. Therapy for lysosomal acid lipase deficiency: replacing a missing link. Hepatology. 2013;58(3):850-852

8. Fasano $T$, Pisciotta L, Bocchi L, Guardamagna O, Assandro $P$ Rabacchi C, et al. Lysosomal lipase deficiency: molecular characterization of eleven patients with Wolman or cholesteryl ester storage disease. Mol Genet Metab. 2012;105(3):450-456.

9. Jones SA, Valayannopoulos V, Schneider E, Eckert S, Banikazemi M Bialer M, et al. Rapid progression and mortality of lysosomal acid lipase deficiency presenting in infants. Genet Med. 2015;18:425-428.

10. Ramírez CM, López AM, Turley SD. Lysosomal acid lipase activity: a tool for the detection and management of fatty liver disease? EBioMedicine, 2015:2:638-639.

11. Camarena C, Aldamiz-Echevarría LJ, Polo B, Barba-Romero MA García I, Cebolla JJ, et al. Actualización en deficiencia de lipasa ácida lisosomal: diagnóstico, tratamiento y seguimiento de los pacientes. Med Clin. 2017;148:429.e1-429.e10.

12. Bay L, Cañero-Velasco C, Ciocca M, Cotti A, Cuarterolo M, Fainboim A, et al. Enfermedad hepática y dislipemia como manifestación de deficiencia de lipasa acida lisosomal (LAL-D). Aspectos clínicos, diagnósticos y nuevo tratamiento. Actualización. Arch Argent Pediatr. 2017;115:287-293.

13. Reynolds T. Cholesteryl ester storage disease: a rare and possibly treatable cause of premature vascular disease and cirrhosis. J Clin Pathol. 2013:66:918-923

14. Scott SA, Liu B, Nazarenko I, Martis S, Kozlitina J, Yang Y, et al. Frequency of the cholesteryl ester storage disease common LIPA E8SJM mutation (c.894G>A) in various racial and ethnic groups. Hepatolology. 2013:58:958-965.

15. Stitziel NO, Fouchier SW, Sjouke B, Peloso GM, Moscoso AM, Auer PL, et al. Exome sequencing and directed clinical phenotyping diagnose cholesterol ester storage disease presenting as autosomal recessive hypercholesterolemia. Arterioscler Thromb Vasc Biol. 2013;33:2909-2914.

16. Muntoni S, Wiebusch H, Jansen-Rust M, Rust S, Seedorf U, Schulte H, et al. Prevalence of cholesteryl ester storage disease. Arterioscler Thromb Vasc Biol. 2007;27:1866-1868.

17. The Human Gene Mutation Database [sitio web]. HGMD gene result. Gales, Reino Unido: Institute of Medical Genetics in Cardiff; 2017.

18. Tanaka A. Acid lipase deficiency: Wolman disease and cholesteryl ester storage disease. Nihon Rinsho. 1995;53:3004-3008.

19. Reference GH [sitio web]. Lysosomal acid lipase deficiency. EE. UU. Genetics Home Reference; 2017.

20. Santillán-Hernández $Y$, Almanza-Miranda E, Xin WW, Goss K, Vera-Loaiza A, Gorráez-De la Mora MT, et al. Novel LIPA mutations in Mexican siblings with lysosomal acid lipase deficiency. World J Gastroenterol. 2015;21:1001-1008.

21. Asociación Española Déficit de Lipasa Ácida Lisosomal [sitio web]. Prevalencia. Déficit de lipasa ácida lisosomal. España: Asociación Española Déficit de Lipasa Ácida Lisosomal; 2019.

22. Valles-Ayoub Y, Esfandiarifard S, No D, Sinai P, Khokher Z, Kohan M, et al. Wolman disease (LIPA p.G87V) genotype frequency in people of Iranian-Jewish ancestry. Genet Test Mol Biomarkers. 2011:15:395-398.

23. Vespasiani-Gentilucci U, Gallo P, Piemonte F, Riva E, Porcari A, Vorini F, et al. Lysosomal acid lipase activity is reduced both in cryptogenic cirrhosis and in cirrhosis of known etiology. PloS One. 2016; 11:e0156113

24. Mendoza-Durán M, Pastor-Rosado J, Fajardo-Giménez M, Vázquez-Pigueiras I, Canals-Candela F, Abad-Linares J. Enfermedad de depósito de ésteres de colesterol. Actualización del diagnóstico y el tratamiento. Acta Pediatr Esp. 2015;73:e31-40

25. Saito S, Ohno K, Suzuki T, Sakuraba H. Structural bases of Wolman disease and cholesteryl ester storage disease. Mol Genet Metab. 2012 Feb;105:244-248.

26. Xanthomatosis G, Calcified W. Glands Early Finding. Reported Pic; 2015

27. Drebber U, Andersen M, Kasper HU, Lohse P, Stolte M, Dienes HP. Severe chronic diarrhea and weight loss in cholesteryl ester storage disease: a case report. World J Gastroenterol. 2005;11:2364-2366.

28. Zhang B, Porto AF. Cholesteryl ester storage disease: protean presentations of lysosomal acid lipase deficiency. J Pediatr Gastroenterol Nutr. 2013;56:682-685

29. Burton BK, Deegan PB, Enns GM, Guardamagna O, Horslen S, Hovingh GK, et al. Clinical features of lysosomal acid lipase deficiency. J Pediatr Gastroenterol Nutr. 2015;61:619-625.
30. Bernstein DL, Hülkova H, Bialer MG, Desnick RJ. Cholesteryl ester storage disease: Review of the findings in 135 reported patients with an underdiagnosed disease. J Hepatol. 2013;58:1230-1243.

31. Morris GE, Braund PS, Moore JS, Samani NJ, Codd V, Webb TR. Coronary artery disease-associated LIPA coding variant rs1051338 reduces lysosomal acid lipase levels and activity in lysosomes. Arterioscler Thromb Vasc Biol. 2017;37:1050-1057.

32. Hůlková $H$, Elleder M. Distinctive histopathological features that support a diagnosis of cholesterol ester storage disease in liver biopsy specimens. Histopathology. 2012;60:1107-1113.

33. Ivashkin V, Zharkova M. Cholesteryl ester crystals in lysosomal acid lipase deficiency. N Engl J Med. 2017;376:e14.

34. Pisciotta L, Fresa R, Bellocchio A, Pino E, Guido V, Cantafora A, et al. Cholesteryl Ester Storage Disease (CESD) due to novel mutations in the LIPA gene. Mol Genet Metab. 2009;97:143-148.

35. Hamilton J, Jones I, Srivastava R, Galloway P. A new method for the measurement of lysosomal acid lipase in dried blood spots using the inhibitor Lalistat 2. Clin Chim Acta. 2012;413:1207-1210.

36. Sreekantam S, Nicklaus-Wollenteit I, Orr J, Sharif K, Vijay S, McKiernan PJ, et al. Successful long-term outcome of liver transplantation in late-onset lysosomal acid lipase deficiency. Pediatr Transplant. 2016:20:851-854.

37. Iverson S, Cairns S, Ward C, Fensom A. Asymptomatic cholesteryl ester storage disease in an adult controlled with simvastatin. Ann Clin Biochem. 1997;34:433-436.

38. Riva S, Spada M, Sciveres M, Minervini M, Cintorino D, Maggiore G, et al. Hepatocarcinoma in a child with cholesterol ester storage disease. Dig Liver Dis. 2008;40:784.

39. Jones S, Bernstein D, Bialer M, Dhawan A, Hendriksz C, Whitley CB, et al. Severe and rapid disease course in the natural history of infants with lysosomal acid lipase deficiency. Mol Genet Metab. 2014; 111:S57-S58.

40. Block RC, Razani B. Options to consider when treating lysosomal acid lipase deficiency. J Clin Lipidol. 2016;10:1280-1281.

41. Balwani M, Breen C, Enns GM, Deegan PB, Honzík T, Jones S, et al. Clinical effect and safety profile of recombinant human lysosomal acid lipase in patients with cholesteryl ester storage disease. Hepatology. 2013;58:950-957.

42. Boletín mensual de la Agencia Española de Medicamentos y Productos Sanitarios junio de 2015. España: AEMPS; 2015.

43. European Medicines Agency. Assessment report. Reino Unido: European Medicines Agency/Committee for Medicinal Products for Human Use; 2015.

44. Rutkowski JV, Burt AD, Leavitt MC, Hu W, Canty D, Quinn AG. Co-localization of macrophage aggregation and fibrosis in a rat model of lysosomal acid lipase (LAL) deficiency and the effects of enzyme replacement with SBC-102. Mol Genet Metab. 2013;108:S80-S81.

45. Rutkowski J, Burt AD, Leavitt M, Hu W, Canty D, Quinn AG. Recombinant human lysosomal acid lipase decreases hepatic macrophage aggregates and colocalized fibrosis in a rat model of lysosomal acid lipase deficiency. hepatology. Hepatology. 2012;56:858A.

46. Thelwall PE, Smith FE, Leavitt MC, Canty D, Hu W, Hollingsworth KG, et al. Hepatic cholesteryl ester accumulation in lysosomal acid lipase deficiency: non-invasive identification and treatment monitoring by magnetic resonance. J Hepatol. 2013;59:543-549.

47. Shirley M. Sebelipase alfa: first global approval. Drugs. 2015;75:1935-1940.

48. Valayannopoulos V, Malinova V, Honzík T, Balwani M, Breen C, Deegan PB, et al. Sebelipase alfa over 52 weeks reduces serum transaminases, liver volume and improves serum lipids in patients with lysosomal acid lipase deficiency. J Hepatol. 2014;61:1135-1142.

49. Hoffman EP, Barr ML, Giovanni MA, Murray MF. Lysosomal acid lipase deficiency. En: Pagon RA, Adam MP, Ardinger HH, Wallace SE, Amemiya A, Bean LJ, et al. , editores. GeneReviews [sitio web]. EE. UU. University of Washington; 1993.

50. Himes RW, Barlow SE, Bove K, Quintanilla NM, Sheridan R, Kohli R. Lysosomal acid lipase deficiency unmasked in two children with nonalcoholic fatty liver disease. Pediatrics. 2016;138:pii.

51. Botero V, García VH, Gómez-Duarte C, Aristizabal AM, Arrunategui AM, Echeverri GJ, et al. Lysosomal acid lipase deficiency, a rare pathology: the first pediatric patient reported in Colombia. Am J Case Rep. 2018;19:669-672. 